\title{
Major Hepatectomy in Elderly Patients with Large Hepatocellular Carcinoma: A Multicenter Retrospective Observational Study
}

This article was published in the following Dove Press journal: Cancer Management and Research

Zheng-Liang Chen, ', Cheng-Wu Zhang, ${ }^{2} *$ Lei Liang, (iD ${ }^{2 * *} \mathrm{Han} \mathrm{Wu}^{3}{ }^{3 *}$ WanGuang Zhang, ${ }^{4}$ Yong-Yi Zeng, ${ }^{5}$ WeiMin Gu, ${ }^{6}$ Ting-Hao Chen, ${ }^{7}$ jie Li, ${ }^{8}$ YaoMing Zhang, ${ }^{9}$ Hong Wang, ${ }^{10}$ YaHao Zhou, ${ }^{\text {" }}$ Chao Li, ${ }^{2}$ Yong-Kang Diao, ${ }^{2}$ Wan Yee Lau, ${ }^{3,12}$ Meng-Chao Wu, ${ }^{3}$ Feng Shen, ${ }^{3}$ Tian Yang, ${ }^{3}$ Ying-Jian Liang (D) 1

'Department of Hepatobiliary Surgery, The First Affiliated Hospital of Harbin Medical University, Heilongjiang, People's Republic of China; 'Department of Hepatobiliary, Pancreatic and Minimal Invasive Surgery, Zhejiang Provincial People's Hospital, People's Hospital of Hangzhou Medical College, Zhejiang, People's Republic of China; ${ }^{3}$ Department of

Hepatobiliary Surgery, Eastern Hepatobiliary Surgery Hospital (Navy Medical University), Second Military Medical University, Shanghai, People's Republic of

China; ${ }^{4}$ Department of Hepatic Surgery, Tongii Hospital, Huazhong University of Science and Technology, Wuhan, People's Republic of China; ${ }^{5}$ Department of Hepatobiliary Surgery, Mengchao Hepatobiliary Hospital, Fujian Medical University, Fujian, People's Republic of China; ${ }^{6}$ The First Department of General Surgery, The Fourth Hospital of Harbin, Heilongjiang, People's Republic of China;

${ }^{7}$ Department of General Surgery, Ziyang First People's Hospital, Sichuan, People's Republic of China; ${ }^{8}$ Department of Hepatobiliary Surgery, Fuyang People's Hospital, Anhui, People's Republic of China; 'The 2nd Department of Hepatobiliary Surgery, Meizhou People's Hospital, Guangdong, People's Republic of China; ${ }^{10}$ Department of General Surgery, Liuyang People's Hospital, Hunan, People's Republic of China;

' 'Department of Hepatobiliary Surgery, Pu'er People's Hospital, Yunnan, People's Republic of China; ${ }^{12}$ Faculty of Medicine, The Chinese University of Hong Kong Shatin, New Territories, Hong Kong, SAR, People's Republic of China

*These authors contributed equally to this work

Correspondence: Ying-Jian Liang

Department of Hepatobiliary Surgery, The First Affiliated Hospital of Harbin Medical University, No. 23, Youzheng Road, Heilongjiang I50006,

People's Republic of China

Email genomeliang@hotmail.com

Tian Yang

Department of Hepatobiliary Surgery, Eastern Hepatobiliary Surgery Hospital, Second Military Medical University (Navy Medical University) No. 225, Changhai Road, Shanghai 200438, People's Republic of China

Email yangtiandfgd@hotmail.com
Background: With an increase in life expectancy and improvement of surgical safety, more elderly patients with hepatocellular carcinoma (HCC), even with large tumors, are now considered for hepatectomy. This study aimed to clarify the impact of age on short- and longterm outcomes after major hepatectomy ( $\geq 3$ segments) for large HCC ( $\geq 5 \mathrm{~cm}$ ).

Patients and Methods: Using a multicenter database, patients who underwent curativeintent major hepatectomy for large HCC between 2006 and 2016 were identified. Postoperative morbidity and mortality, overall survival (OS) and recurrence-free survival (RFS) were compared between the elderly ( $\geq 65$ years) and younger ( $<65$ years) patients. Univariable and multivariable Cox-regression analyses were performed to identify the risk factors of OS and RFS in the entire and elderly cohorts, respectively.

Results: Of 830 patients, 92 (11.1\%) and 738 (88.9\%) were elderly and younger patients, respectively. There were no significant differences in postoperative 30-day mortality and morbidity between the two groups ( $5.4 \%$ vs $2.6 \%$ and $43.5 \%$ vs $38.3 \%$, both $\mathrm{P}>0.05$ ). The 5 -year OS and RFS rates in elderly patients were also comparable to younger patients (35.0\% vs $33.2 \%$ and $20.0 \%$ vs $20.8 \%$, both $\mathrm{P}>0.05$ ). In the entire cohort, multivariable Cox-regression analyses identified that old age was not independently associated with OS and RFS. However, in the elderly cohort, preoperative alpha-fetoprotein level $>400 \mu \mathrm{g} / \mathrm{L}$, multiple tumors, macrovascular invasion and microvascular invasion were independently associated with decreased OS and RFS. Conclusion: Carefully selected elderly patients benefited from major hepatectomy for large $\mathrm{HCC}$ as much as younger patients, and their long-term prognosis was determined by preoperative alpha-fetoprotein level, tumor number and presence of macro- or microvascular invasion.

Keywords: hepatocellular carcinoma, hepatectomy, survival, recurrence, elderly

\section{Introduction}

Hepatocellular carcinoma (HCC) is the fourth leading cause of cancer-related mortality in adults, ${ }^{1}$ and is the most common cancer affecting the elderly worldwide. ${ }^{2}$ The overall prognosis of HCC remains dismal. For a subset of patients receiving curative therapies including local ablation, liver transplantation and partial hepatectomy, ${ }^{3}$ long-term survival is still possible. Local ablation, albeit less invasive, is only suitable for small $\mathrm{HCC}$, generally considered to be less than $5 \mathrm{~cm}$. Liver transplantation is the treatment of choice for early HCC that meets the Milan criteria. However, the lack of donors limits its application in elderly patients, especially in Asia. Thus, for patients with a large $\mathrm{HCC}(\geq 5 \mathrm{~cm})$, especially in elderly patients, the only possible cure is partial hepatectomy. 
Advances in surgical techniques, perioperative assessments, anesthesia and medical care have led to notably decrease in postoperative morbidity and mortality following hepatectomy for HCC. In general, selected elderly patients, although having more associated comorbidities and compromised organ reserve, can still well tolerate hepatectomy for HCC with short- and long-term outcomes similar to younger patients. Age by itself did not have any significant negative impact. ${ }^{4-10}$ Major hepatectomy $(\geq 3$ Couinaud segments) for large HCC intrinsically has a higher surgical risk. ${ }^{11}$ However, so far there have been few studies on whether this operation in elderly patients is safe, efficacious and having long-term oncological outcomes similar to younger patients. ${ }^{12,13}$

The aim of the present study was to determine safety and efficacy in elderly patients undergoing major hepatectomy for large HCC using a large multicenter database. The short- and long-term outcomes, including long-term overall (OS) and recurrence-free survival (RFS), in elderly patients $(\geq 65$ years old) were compared with younger patients $(<65$ years old).

\section{Patients and Methods Study Population}

Using a multicenter database, patients who underwent curative-intent hepatectomy for HCC at 11 medical institutions in China from January 2006 to December 2016 were identified. These institutions included the First Affiliated Hospital of Harbin Medical University, Zhejiang Provincial People's Hospital, Eastern Hepatobiliary Surgery Hospital, Tongji Hospital, Mengchao Hepatobiliary Hospital, the Fourth Hospital of Harbin, Ziyang First People's Hospital, Fuyang People's Hospital, Meizhou People's Hospital, Liuyang People's Hospital, and Pu'er People's Hospital. Major hepatectomy was defined as partial hepatectomy of three or more Couinaud's segments. Curative hepatectomy was defined as the removal of all microscopic and macroscopic tumors with a microscopically clear margin (R0 resection). The exclusion criteria included: 1) patient $\leq 18$ years old, 2) combined HCCcholangiocarcinoma, 3) recurrent HCC, 4) palliative hepatectomy (R1 or R2 resection), 5) HCC with a maximum tumor size $<5 \mathrm{~cm}, 6$ ) minor hepatectomy ( $<3$ segments), 7) loss to follow-up within 3 months after surgery, and 8) missing data on important prognostic variables. Informed consent was obtained from all the patients for their data to be used for clinical research. The data were censored on June 30, 2019. The study was performed according to the Declaration of
Helsinki and the Ethical Guidelines for Clinical Studies. Approval from Ethics Committees of each of the individual hospital was obtained.

\section{Preoperative Evaluation}

Preoperative evaluation was generally consistent at each participating institution. Liver function was evaluated by biochemistry and Child-Pugh classification. Upper gastrointestinal endoscopy was routinely employed for all HCC patients who were scheduled for operation. Patients older than 60 years and those with significant co-morbid illnesses were routinely sent for formal cardiopulmonary evaluation. ${ }^{14,15}$ Child-Pugh grade $\mathrm{C}$ was considered an absolute contraindication for $\mathrm{HCC}$ resection. Resection criteria were constant over the study period, including the number of resectable tumors, presence or absence of gross tumor thrombus, and adequate liver function reserve, as reported in our previous study. ${ }^{14,16}$

\section{Clinicopathologic Characteristics and Operative Variables}

The following patients' clinicopathologic characteristics were collected, including: age, sex, co-morbidities, American Society of Anesthesiologists (ASA) score, etiology of liver diseases, presence of cirrhosis or portal hypertension, ChildPugh grading, preoperative alpha-fetoprotein (AFP) level, maximum tumor size, tumor number, presence of satellite nodules, tumor differentiation, presence of macroscopic or microscopic vascular invasion, tumor encapsulation, and resection margin status. Co-morbidities included hypertension, diabetes mellitus, chronic obstructive pulmonary disease, renal dysfunction, and cardiovascular disease. Cirrhosis was confirmed by histopathological examination. Portal hypertension was defined as the presence of either splenomegaly with a decreased platelet count $\left(\leq 100 \times 10^{9} / \mathrm{L}\right)$ or esophageal varices. Operative variables collected included intraoperative blood loss and intraoperative blood transfusion.

\section{Follow-Up}

Patients were followed-up at each participating institution. The routine postoperative surveillance strategy for recurrence included serum AFP level, ultrasonography or contrast-enhanced computed tomography (CT) or magnetic resonance imaging (MRI) of the chest and abdomen once every 2 months for the first 6 months, once every 3 months thereafter for the next 18 months, and then once every 6 months at 2 years and later after resection. When HCC 
recurrence was suspected, contrast-enhanced CT or MRI, chest $\mathrm{CT}$, bone scan or positron emission tomography were performed as clinically indicated. Tumor recurrence was defined as a new appearance of intra- or extra-hepatic tumor nodule(s) with or without a rise in serum AFP level, as well as the presence of intrahepatic nodules that had typical imaging features with characteristic features of HCC on contrast-enhanced CT or MRI examination. Treatment of HCC recurrence was based on the pattern of recurrence, residual hepatic functional reserve, and general condition of patient.

\section{Study Endpoints}

As the present study focused on perioperative safety and long-term survival outcomes of hepatectomy for HCC, the primary endpoints were OS and RFS, while the secondary endpoints were postoperative 30-day mortality and morbidity. OS was defined as the time from surgery to death from any cause, and RFS as the time from surgery to HCC recurrence or death. Postoperative morbidities were classified into five grades based on the Clavien-Dindo classification, ${ }^{17}$ and minor and major morbidities were defined as Clavien-Dindo $<3$ and $\geq 3$, respectively.

\section{Statistical Analysis}

Statistical analyses were performed using SPSS software version 25.0 (SPSS, Chicago, IL, USA). Categorical variables were expressed as number (n) or proportion (\%), while continuous variables were expressed as mean \pm standard deviation or median (range). Categorical and continuous covariates were compared using the $\chi^{2}$ test with Yates correction or Fisher's exact test, and Student's $t$ test or Mann-Whitney ranked $U$-test, respectively. The OS and RFS rates were estimated using the Kaplan-Meier curves and compared with the Log-rank test. Univariable and Multivariable Cox-regression analyses were performed to identify risk factors which were independently associated with decreased OS and RFS in the entire cohort, as well as in the elderly cohort. The variable of age, as the most important variable, was forced into the multivariable Cox-regression model. Other variables with a $P$ value $<0.1$ in univariable analysis were subjected to the multivariable Cox-regression model using a forward stepwise selection. All tests were two-tailed, and a significant $P$ value was defined as $<0.05$.

\section{Results}

The multicenter database contained details of 2133 patients from 11 institutions who underwent partial hepatectomy with the curative intention for HCC during the study period. Using the inclusion criteria, 830 patients who underwent curative major hepatectomy for large HCC were enrolled in this study (Figure 1). Among all the 830 patients identified in the entire cohort, there were 92 elderly patients ( $\geq 65$ years old, $11.1 \%$ ) and 738 younger patients ( $<65$ years old, $88.9 \%$ ), with a median age of 68 (range: 65 to 83) and 49 (range: 21 to 64) years, respectively. Patients' clinicopathologic characteristics and operative variables among the elderly versus the younger were notable for several differences (Table 1): proportion of male patients, patients with co-morbidities, ASA score $>2$, chronic hepatitis B virus (HBV) infection and satellite nodules were significantly higher in the elderly group (all $P<0.05$ ).

\section{Comparisons of Postoperative Morbidity and Mortality}

Postoperative short-term outcomes of the elderly and younger patient groups are summarized in Table 1. The postoperative 30-day mortality and morbidity rates were comparable in the two groups $(5.443 .5 \%$ vs $2.6 \%$ and $38.3 \%, P=0.173$ and 0.365 , respectively). The postoperative major and minor morbidity rates were also comparable $(17.426 .1 \%$ vs $17.6 \%$ and $20.7 \%, \mathrm{P}=1.000$ and 0.280 , respectively). Univariable and multivariable logistic regression analyses of risk factors associated with postoperative 30-day morbidity are shown in Table 2.

\section{Comparisons of Long-Term Survival and HCC Recurrence}

After excluding 24 patients who suffered from postoperative 30-day death, long-term outcomes of the remaining 806 patients were compared between the two groups (Table 3). At a median follow-up of 26.4 months (mean follow-up: 36.8 months), 569 of 806 patients $(70.6 \%)$ had died and 622 (77.2\%) had developed HCC recurrence. The overall mortality and recurrence rates among the elderly versus the younger groups were 72.4 and $70.1 \%$ versus 77.7 and $70.7 \%$, respectively (both $P>0.2$ ). The median OS and RFS in the elderly group were 30.6 and 9.8 months, while the median OS and RFS in the younger group were 25.6 and 8.8 months, and there was no significant difference between these two groups $(P=0.739$ and 0.632 , respectively). As 


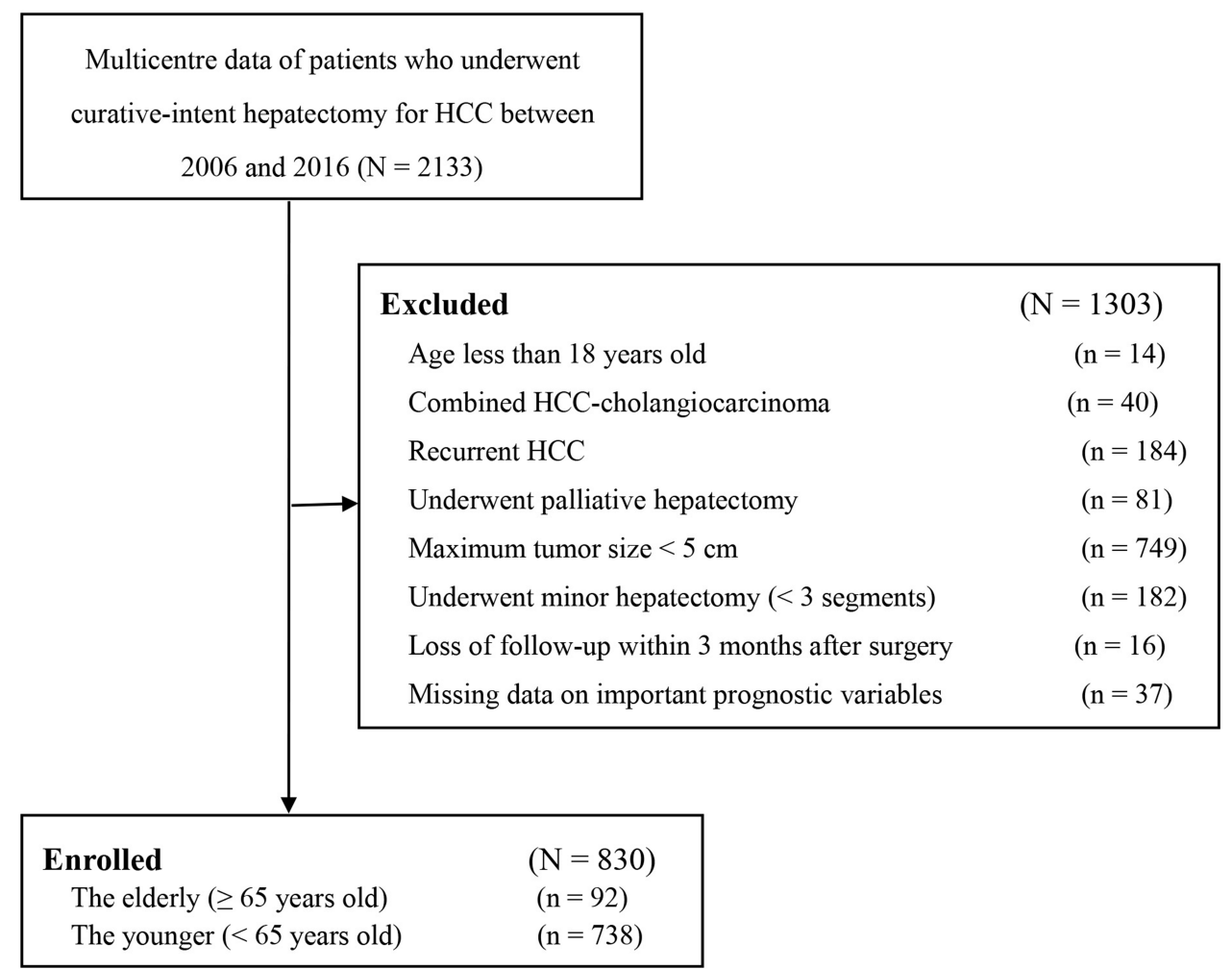

Figure I Flowchart of study population. HCC, hepatocellular carcinoma.

shown in Table 3 and Figure 2, the 1-, 3- and 5-year OS and RFS rates in the elderly group were $71.3 \%, 43.2 \%$ and $35.0 \%$, and $41.4 \%, 27.3 \%$ and $20.0 \%$, respectively, which were comparable to the younger group $(68.9 \%, 42.0 \%$ and $33.2 \%$, and $44.0 \%, 28.3 \%$ and $20.8 \%$, respectively).

\section{Analyses of Prognostic Factors for OS and RFS in the Entire Cohort}

Independent risk factors associated with OS and RFS after major hepatectomy for large HCC were identified by univariable and multivariable Cox-regression analyses (Tables 4 and 5). Multivariable analyses demonstrated that old age was not independently associated with OS and RFS (both $\mathrm{P}>0.2$ ), but preoperative AFP level $>400 \mu \mathrm{g} / \mathrm{L}$ (hazard ratio [HR] 1.53, 95\% confidence interval [CI] 1.29-1.82), satellite nodules (HR 1.75, 95\% CI 1.48-2.08), macrovascular invasion (HR 2.48, 95\% CI 2.06-2.99), microvascular invasion (HR 2.30, 95\% CI 1.91-2.76), and intraoperative blood transfusion (HR 2.34, 95\% CI 1.13-1.58) were independent risk factors of decreased OS, while preoperative AFP level $>400 \mu \mathrm{g} / \mathrm{L}$ (HR 1.54, 95\% CI 1.31-1.81), satellite nodules (HR 1.51, 95\% CI 1.28-1.78), macrovascular invasion (HR 2.14, 95\% CI 1.76-2.46), microvascular invasion (HR 1.62, 95\% CI 1.34-1.95), intraoperative blood transfusion (HR 1.27, 95\% CI 1.08-1.49), and resection margin $<1 \mathrm{~cm}$ (HR 2.08, 95\% CI 1.76-2.46) were independent risk factors of decreased RFS after major hepatectomy for large HCC.

\section{Prognostic Analyses for OS and RFS in the Elderly Cohort}

Table 6 lists the risk factors identified by univariable and multivariable Cox-regression analyses which were associated with OS after major hepatectomy for HCC in the elderly cohort. Multivariable analyses demonstrated that preoperative AFP level $>400 \mu \mathrm{g} / \mathrm{L}$ (HR 1.84, 95\% CI 1.04-3.26), multiple tumors ( $\geq 3$ tumor nodules) (HR $2.07,95 \%$ CI 1.18-3.62), and macrovascular invasion (HR 3.01, 95\% CI 1.58-5.74) were independent risk factors of decreased OS after major hepatectomy for elderly patients with a large HCC.

Table 7 lists the risk factors identified by univariable and multivariable Cox-regression analysis which were associated with RFS after major hepatectomy for HCC in the elderly cohort. Multivariable analyses identified preoperative AFP level $>400 \mu \mathrm{g} / \mathrm{L}$ (HR 1.82, 95\% CI 1.03-3.22), multiple tumors (HR 2.47, 95\% CI 1.37-4.44), macrovascular invasion (HR 2.71, 95\% CI 1.54-4.77), and 
Table I Comparisons of Clinicopathologic Characteristics, Operative Variables and Short-Term Outcomes Following Major Hepatectomy for Large Hepatocellular Carcinoma Between Elderly and Younger Patients in the Entire Cohort

\begin{tabular}{|c|c|c|c|}
\hline $\mathbf{N}(\%)$ & The Younger $(\mathbf{N}=738)$ & The Elderly ( $\mathrm{N}=92)$ & $P$ \\
\hline \multicolumn{4}{|l|}{ Clinicopathologic characteristics } \\
\hline Age, years* & $47.6 \pm 9.2$ & $69.0 \pm 4.0$ & $<0.001$ \\
\hline Male sex & $654(88.6)$ & $73(79.3)$ & 0.018 \\
\hline Co-morbidities & $108(14.6)$ & 31 (33.7) & $<0.001$ \\
\hline ASA score $>2$ & $75(10.2)$ & $39(42.4)$ & $<0.001$ \\
\hline \multicolumn{4}{|l|}{ Etiology of liver diseases } \\
\hline HBV & $672(91.0)$ & $63(68.5)$ & $<0.001$ \\
\hline $\mathrm{HCV}$ & $5(0.7)$ & $2(2.2)$ & \\
\hline $\mathrm{HBV}+\mathrm{HCV}$ & $4(0.5)$ & $2(2.2)$ & \\
\hline Others** & $57(7.7)$ & $25(27.2)$ & \\
\hline Cirrhosis & $534(72.4)$ & $59(64.1)$ & 0.111 \\
\hline Portal hypertension & $124(16.8)$ & $14(15.2)$ & 0.768 \\
\hline Child-Pugh grade B & $79(10.7)$ & $8(8.7)$ & 0.718 \\
\hline Preoperative AFP level > 400 ug/L & $308(4 I .7)$ & $30(32.6)$ & 0.115 \\
\hline Maximum tumor size, $\mathrm{cm}^{*}$ & $10.8 \pm 3.3$ & $10.5 \pm 3.8$ & 0.474 \\
\hline Maximum tumor size $\geq 10 \mathrm{~cm}$ & $430(58.3)$ & $49(53.3)$ & 0.372 \\
\hline Multiple tumors ( $\geq 3$ tumor nodules) & $145(19.6)$ & 14 (I5.2) & 0.399 \\
\hline Satellite nodules & $290(39.3)$ & $25(27.2)$ & 0.030 \\
\hline Poor tumor differentiation & $540(73.2)$ & $63(68.5)$ & 0.385 \\
\hline Macrovascular invasion & $189(25.6)$ & $25(27.2)$ & 0.800 \\
\hline Microvascular invasion & $475(64.4)$ & $52(56.5)$ & 0.168 \\
\hline Incomplete tumor encapsulation & $525(7 I .1)$ & $60(65.2)$ & 0.275 \\
\hline Resection margin $<\mathrm{I} \mathrm{cm}$ & $260(35.9)$ & $33(35.9)$ & 1.000 \\
\hline \multicolumn{4}{|l|}{ Operative variables } \\
\hline Intraoperative blood loss, mL* & $500(50-8000)$ & $550(100-4300)$ & 0.235 \\
\hline Intraoperative blood transfusion & $260(35.2)$ & $4 \mathrm{I}(44.6)$ & 0.085 \\
\hline \multicolumn{4}{|l|}{ Short-term outcomes } \\
\hline Postoperative hospital stay* & $13.6 \pm 6.3$ & $14.6 \pm 7.8$ & 0.178 \\
\hline Postoperative 30-day mortality & $19(2.6)$ & $5(5.4)$ & 0.173 \\
\hline Acute liver failure & 10 & 2 & \\
\hline Abdominal hemorrhage & 3 & 1 & \\
\hline Pulmonary infection & 3 & 1 & \\
\hline Cardiovascular accident & I & 1 & \\
\hline Upper gastrointestinal bleeding & I & 0 & \\
\hline Pulmonary embolism & I & 0 & \\
\hline Postoperative 30-day morbidity & $283(38.3)$ & $40(43.5)$ & 0.365 \\
\hline Postoperative 30 -day major morbidity & $130(17.6)$ & $16(17.4)$ & 1.000 \\
\hline Postoperative 30 -day minor morbidity & $153(20.7)$ & $24(26.1)$ & 0.280 \\
\hline
\end{tabular}

Notes: *Values are mean \pm standard deviation or median with range. **Others include alcoholic liver disease, non-alcoholic fatty liver disease, schistosomiasis cirrhosis, and cryptogenic cirrhosis.

Abbreviations: ASA, American Society of Anesthesiologists; HBV, hepatitis B virus; HCV, hepatitis C virus; AFP, alpha-fetoprotein.

microvascular invasion (HR 1.85, 95\% CI 1.06-3.21) to be independent risk factors of decreased RFS after major hepatectomy for elderly patients with a large HCC.

According to the number of three independent risk factors which could be identified before surgery, ie preoperative AFP level $>400 \mu \mathrm{g} / \mathrm{L}$, the presence of macrovascular invasion, and multiple tumor ( $\geq 3$ nodules), the 87 elderly patients were divided into 3 subgroups: 1) without any risk $(\mathrm{n}=43) ; 2)$ with any one of the three risks $(\mathrm{n}=30)$; and 3$)$ with $\geq 2$ of the three risks ( $n=14 ; 10$ patients with any 2 of the three risks and 4 patients with all three risks). As shown in Figure 3, there were significant differences between OS 
Table 2 Univariable and Multivariable Logistic Regression Analyses of Risk Factors of Postoperative 30-Day Morbidity After Major Hepatectomy for Large Hepatocellular Carcinoma in the Entire Cohort

\begin{tabular}{|c|c|c|c|c|c|}
\hline Variables & OR Comparison & UV OR $(95 \% \mathrm{CI})$ & UV $P$ & MV OR $(95 \% \mathrm{CI})$ & MV P* \\
\hline Age & Elderly vs Younger & $1.16(0.74-1.83)$ & 0.365 & NA & 0.596 \\
\hline Sex & Male vs Female & $1.14(0.74-1.75)$ & 0.555 & & \\
\hline Co-morbidities & Yes vs No & $1.84(0.95-3.57)$ & 0.070 & NA & 0.148 \\
\hline ASA score & $>2$ vs $\leq 2$ & $1.73(\mathrm{I} .15-2.6 \mathrm{I})$ & 0.009 & NA & 0.072 \\
\hline Etiology of liver diseases & HBV vs Non-HBV & $1.16(0.72-1.86)$ & 0.548 & & \\
\hline Cirrhosis & Yes vs No & $2.13(1.52-2.98)$ & $<0.001$ & $2.03(1.44-2.88)$ & $<0.001$ \\
\hline Portal hypertension & Yes vs No & $1.60(1.10-2.33)$ & 0.015 & NA & 0.482 \\
\hline Child-Pugh grade & $B$ vs $A$ & $2.20(1.37-3.54)$ & 0.001 & $1.74(1.06-2.83)$ & 0.027 \\
\hline Preoperative AFP level & $>400$ vs $\leq 400 \mu \mathrm{g} / \mathrm{L}$ & $1.20(0.90-1.59)$ & 0.221 & & \\
\hline Maximum tumor size & $\geq 10$ vs $5.0-9.9 \mathrm{~cm}$ & $1.26(0.94-1.68)$ & 0.121 & & \\
\hline Multiple tumors ( $\geq 3$ nodules) & Yes vs No & $1.51(1.03-2.20)$ & 0.034 & $1.67(1.13-2.48)$ & 0.011 \\
\hline Satellite nodules & Yes vs No & $0.95(0.7 \mathrm{I}-\mathrm{I} .28)$ & 0.747 & & \\
\hline Poor tumor differentiation & Yes vs No & $0.78(0.57-1.07)$ & 0.131 & & \\
\hline Macrovascular invasion & Yes vs No & $1.42(1.08-1.97)$ & 0.033 & NA & 0.086 \\
\hline Microvascular invasion & Yes vs No & $1.4 \mid(1.05-1.89)$ & 0.024 & NA & 0.169 \\
\hline Incomplete tumor encapsulation & Yes vs No & $1.28(0.93-1.75)$ & $0.13 \mid$ & & \\
\hline Intraoperative blood loss & $>600$ vs $\leq 600 \mathrm{~mL}$ & $1.96(1.46-2.64)$ & $<0.001$ & NA & 0.256 \\
\hline Intraoperative blood transfusion & Yes vs No & $2.10(1.56-2.82)$ & $<0.001$ & $2.05(1.5 \mathrm{I}-2.78)$ & $<0.001$ \\
\hline Resection margin & $<\mathrm{I} v \mathrm{vs} \geq \mathrm{I} \mathrm{cm}$ & $1.17(0.84-1.64)$ & 0.349 & & \\
\hline
\end{tabular}

Notes: *As the most important variable, age was forced into multivariable logistic regression model; apart from age, other variables with a $P$ value $<0.1$ in univariable analysis were subjected to multivariable logistic regression model.

Abbreviations: ASA, American Society of Anesthesiologists; HBV, hepatitis B virus; AFP, alpha-fetoprotein; Cl, confidence interval; OR, odds ratio; UV, univariable; MV, multivariable; NA, not available.

Table 3 Comparisons of Long-Term Outcomes Following Major Hepatectomy for Large Hepatocellular Carcinoma Between Elderly and Younger Patients in the Entire Cohort of the Remaining 806 Patients After Excluding 24 Patients Who Died Within 30 Days After Surgery

\begin{tabular}{|l|l|l|l|}
\hline $\mathbf{N}$ (\%) & The Younger $\mathbf{( N = 7 1 9 )}$ & The Elderly $\mathbf{( N = 8 7 )}$ & $\mathbf{P}$ \\
\hline Recurrence during the follow-up & $559(77.7)$ & $63(72.4)$ & 0.280 \\
Intrahepatic recurrence & $500(89.4)$ & $52(82.5)$ & \\
Extrahepatic recurrence & $17(3.0)$ & $3(4.8)$ & \\
Intra- and extrahepatic recurrence & $42(7.5)$ & $61(70.1)$ & 0.901 \\
\hline Death during the follow-up & $508(70.7)$ & $30.6(22.0-39.2)$ & 0.739 \\
\hline Median OS, 95\% Cl & $25.6(21.4-29.8)$ & 71.3 & \\
I-year OS rate, \% & 68.9 & 43.2 & \\
3-year OS rate, \% & 42.0 & 35.0 & 0.632 \\
5-year OS rate, \% & 33.2 & $9.8(6.3-13.3)$ & \\
\hline Median RFS, 95\% Cl & $8.8(7.2-10.4)$ & 41.4 & \\
I-year RFS rate, \% & 44.0 & 27.3 & \\
3-year RFS rate, \% & 28.3 & 20.0 & \\
5-year RFS rate, \% & 20.8 & & \\
\hline
\end{tabular}

Abbreviations: OS, overall survival; RFS, recurrence-free survival; $\mathrm{Cl}$, confidence interval.

and RFS among these three subgroups (both $P<0.001$ ). Of note, the 1-year OS and RFS rates of those 14 patients who had any 2 of the three risks were only $35.7 \%$ and $14.3 \%$, respectively.

\section{Discussion}

With aging populations, prolonged life expectancy, and improvements in perioperative management, the number of elderly patients undergoing hepatectomy for $\mathrm{HCC}$ in 

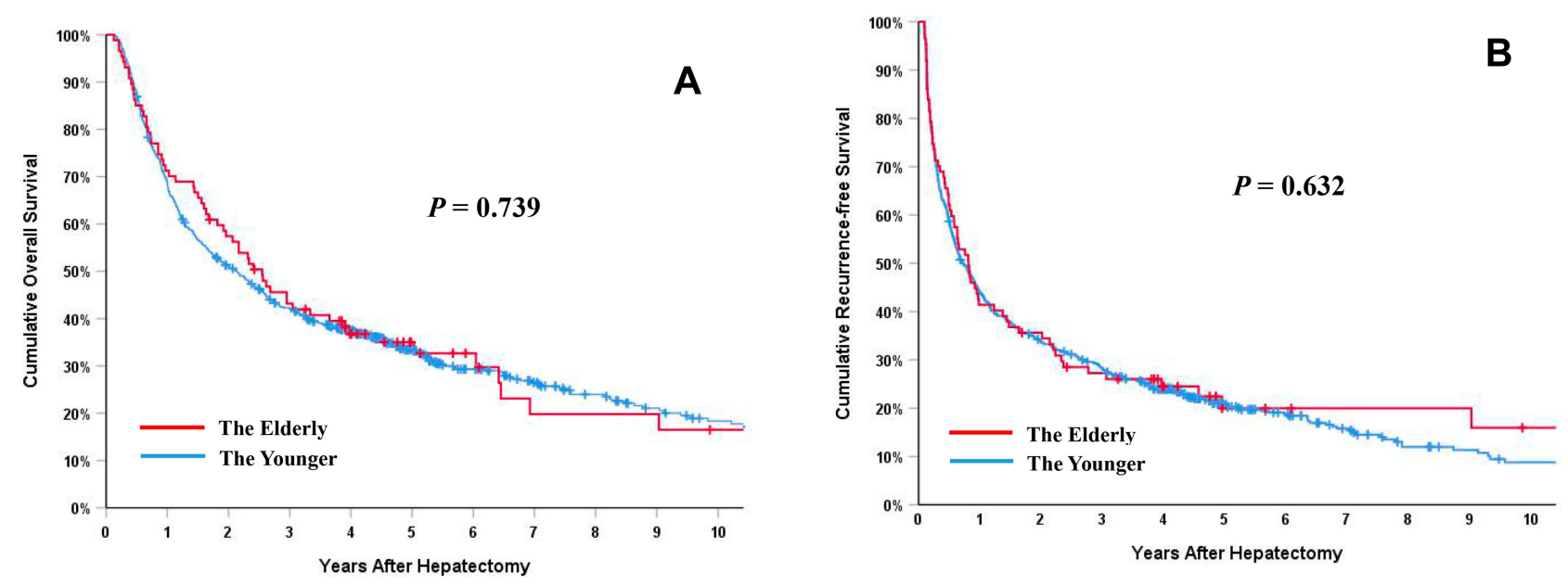

Figure 2 Cumulative incidence of overall survival (OS, 2A) and recurrence-free survival (RFS, 2B) curves comparisons between elderly and younger patients (calculated by Log-rank test).

centers around the world has significantly increased in the past three decades. ${ }^{5-7,10,18,19}$ However, the safety and effectiveness of major hepatectomy ( $\geq 3$ segments) for large HCC ( $\geq 5 \mathrm{~cm})$ among elderly patients have still not been well defined. ${ }^{20-22}$ There are several major areas of concern: 1) more comorbidities and compromised organ functional reserve in the elderly; 2) more complicated surgical procedure resulting in higher perioperative risks in major hepatectomy; and 3) uncertain long-term survival outcomes for large HCC.

This large multicenter study of 830 patients with large HCC revealed that major hepatectomy can safely be performed in the elderly ( $\geq 65$ years old), with postoperative morbidity and mortality rates similar to younger patients

Table 4 Univariable and Multivariable Cox-Regression Analyses of Risk Factors of Overall Survival After Major Hepatectomy for Large Hepatocellular Carcinoma in the Entire Cohort of the Remaining 806 Patients After Excluding 24 Patients Who Died Within 30 Days After Surgery

\begin{tabular}{|c|c|c|c|c|c|}
\hline Variables & HR Comparison & UV HR (95\% CI) & UV P & MV HR (95\% Cl) & MV P* \\
\hline Age & Elderly vs Younger & $0.96(0.73-1.25)$ & 0.739 & NA & 0.676 \\
\hline Sex & Male vs Female & $0.99(0.77-1.27)$ & 0.918 & & \\
\hline Co-morbidities & Yes vs No & $1.21(0.82-1.80)$ & 0.340 & & \\
\hline ASA score & $>2$ vs $\leq 2$ & $1.07(0.84-1.36)$ & 0.573 & & \\
\hline Etiology of liver diseases & HBV vs Non-HBV & I.29 (0.97-I.7I) & 0.078 & NA & 0.414 \\
\hline Cirrhosis & Yes vs No & $1.29(1.07-1.56)$ & 0.007 & NA & 0.436 \\
\hline Portal hypertension & Yes vs No & $1.49(1.21-1.83)$ & $<0.001$ & NA & 0.207 \\
\hline Child-Pugh grade & $B$ vs $A$ & $1.23(0.94-1.61)$ & 0.139 & & \\
\hline Preoperative AFP level & $>400$ vs $\leq 400 \mu \mathrm{g} / \mathrm{L}$ & $1.79(1.51-2.12)$ & $<0.001$ & $1.53(1.29-1.82)$ & $<0.001$ \\
\hline Maximum tumor size & $\geq 10$ vs $5.0-9.9 \mathrm{~cm}$ & $1.23(1.04-1.46)$ & 0.015 & NA & 0.673 \\
\hline Multiple tumors ( $\geq 3$ nodules) & Yes vs No & $1.93(1.58-2.34)$ & $<0.001$ & NA & 0.147 \\
\hline Satellite nodules & Yes vs No & $2.09(1.77-2.47)$ & $<0.001$ & $1.75(1.48-2.08)$ & $<0.001$ \\
\hline Poor tumor differentiation & Yes vs No & $0.96(0.80-1.16)$ & 0.686 & & \\
\hline Macrovascular invasion & Yes vs No & $2.97(2.48-3.56)$ & $<0.001$ & $2.48(2.06-2.99)$ & $<0.001$ \\
\hline Microvascular invasion & Yes vs No & $1.68(I .4 I-2.01)$ & $<0.001$ & $2.30(1.91-2.76)$ & $<0.001$ \\
\hline Incomplete tumor encapsulation & Yes vs No & $2.20(1.81-2.69)$ & $<0.001$ & NA & 0.214 \\
\hline Intraoperative blood loss & $>600$ vs $\leq 600 \mathrm{~mL}$ & $1.46(1.24-1.73)$ & $<0.001$ & NA & 0.725 \\
\hline Intraoperative blood transfusion & Yes vs No & $1.47(1.24-1.74)$ & $<0.001$ & $2.34(1.13-1.58)$ & 0.001 \\
\hline Resection margin & $<\mathrm{I} v \mathrm{vs} \geq \mathrm{I} \mathrm{cm}$ & $2.30(1.91-2.77)$ & $<0.001$ & NA & 0.062 \\
\hline
\end{tabular}

Notes: *As the most important variable, age was forced into multivariable Cox-regression model; apart from age, other variables with a $P$ value $<0 . I$ in univariable analysis were subjected to multivariable Cox-regression model.

Abbreviations: ASA, American Society of Anesthesiologists; HBV, hepatitis B virus; AFP, alpha-fetoprotein; Cl, confidence interval; HR, hazard ratio; UV, univariable; MV, multivariable; NA, not available. 
Table 5 Univariable and Multivariable Cox-Regression Analyses of Risk Factors of Recurrence-Free Survival After Major Hepatectomy for Large Hepatocellular Carcinoma in the Entire Cohort of the Remaining 806 Patients After Excluding 24 Patients Who Died Within 30 Days After Surgery

\begin{tabular}{|c|c|c|c|c|c|}
\hline Variables & HR Comparison & UV HR (95\% Cl) & UV P & MV HR (95\% Cl) & $M V P *$ \\
\hline Age & Elderly vs Younger & $0.94(0.73-1.21)$ & 0.632 & NA & 0.266 \\
\hline Sex & Male vs Female & $1.06(0.84-1.33)$ & 0.646 & & \\
\hline Co-morbidities & Yes vs No & $1.09(0.75-1.57)$ & 0.655 & & \\
\hline ASA score & $>2$ vs $\leq 2$ & $0.98(0.78-1.23)$ & 0.851 & & \\
\hline Etiology of liver diseases & HBV vs Non-HBV & $1.45(1.12-1.89)$ & 0.006 & NA & 0.891 \\
\hline Cirrhosis & Yes vs No & $1.23(1.03-1.46)$ & 0.019 & NA & 0.606 \\
\hline Portal hypertension & Yes vs No & $1.36(1.12-1.66)$ & 0.002 & NA & 0.222 \\
\hline Child-Pugh grade & $B$ vs $A$ & $\mathrm{I} .42(\mathrm{I} . \mathrm{II}-\mathrm{I} .82)$ & 0.005 & NA & 0.202 \\
\hline Preoperative AFP level & $>400$ vs $\leq 400 \mu g / L$ & $1.80(1.54-2.11)$ & $<0.001$ & $1.54(1.31-1.81)$ & $<0.001$ \\
\hline Maximum tumor size & $\geq 10$ vs $5.0-9.9 \mathrm{~cm}$ & $1.24(1.06-1.45)$ & 0.006 & NA & 0.806 \\
\hline Multiple tumors ( $\geq 3$ nodules) & Yes vs No & $1.79(1.48-2.16)$ & $<0.001$ & NA & 0.276 \\
\hline Satellite nodules & Yes vs No & $1.92(1.64-2.24)$ & $<0.001$ & $1.5 \mathrm{I}(1.28-1.78)$ & $<0.001$ \\
\hline Poor tumor differentiation & Yes vs No & $1.16(0.97-1.39)$ & 0.098 & NA & 0.745 \\
\hline Macrovascular invasion & Yes vs No & $2.82(2.37-3.35)$ & $<0.001$ & $2.14(1.76-2.46)$ & $<0.001$ \\
\hline Microvascular invasion & Yes vs No & $1.69(1.43-1.99)$ & $<0.001$ & $1.62(1.34-1.95)$ & $<0.001$ \\
\hline Incomplete tumor encapsulation & Yes vs No & $2.00(1.68-2.39)$ & $<0.001$ & NA & 0.179 \\
\hline Intraoperative blood loss & $>600$ vs $\leq 600 \mathrm{~mL}$ & $1.46(|.25-| .7 I)$ & $<0.001$ & NA & 0.706 \\
\hline Intraoperative blood transfusion & Yes vs No & $1.42(1.22-1.67)$ & $<0.001$ & $1.27(1.08-1.49)$ & 0.003 \\
\hline Resection margin & $<\mathrm{I} v \mathrm{vs} \geq \mathrm{I} \mathrm{cm}$ & $2.00(1.69-2.36)$ & $<0.001$ & $2.08(1.76-2.46)$ & $<0.001$ \\
\hline
\end{tabular}

Notes: *As the most important variable, age was forced into multivariable Cox-regression model; apart from age, other variables with a $P$ value $<0.1$ in univariable analysis were subjected to multivariable Cox-regression model.

Abbreviations: ASA, American Society of Anesthesiologists; HBV, hepatitis B virus; AFP, alpha-fetoprotein; Cl, confidence interval; HR, hazard ratio; UV, univariable; MV, multivariable; NA, not available.

of $<65$ years old; moreover, elderly patients can benefit from major hepatectomy for large HCC, with long-term OS and RFS outcomes similar to younger patients. This study supports the findings that age by itself is not a contraindication to major hepatectomy of large HCC, with oncological benefits and an acceptable safety profile. Major hepatectomy should be offered to selected elderly patients with large HCC. The present study on analyzing the prognostic factors of OS and RFS in the elderly cohort demonstrated that preoperative AFP level $>400 \mu \mathrm{g} / \mathrm{L}$, multiple tumors ( $\geq 3$ tumor nodules) and macrovascular invasion were independently associated with decreased OS and RFS in elderly patients after major hepatectomy for large HCC. These data are helpful in the surgical decisionmaking in selecting patients for surgery and in assessing whether adjuvant therapy should be considered for elderly patients with large HCC. To our knowledge, this is the first study to evaluate specifically surgical safety and long-term survival outcomes in elderly patients who underwent major hepatectomy for large HCC. The results from this Multicenter cooperative study may lead to a more widely accepted consensus on the role of major hepatectomy in elderly patients with large HCC.
Malignancies are often considered to be a disease associated with aging. ${ }^{23,24}$ The probability of developing a malignant tumor in the elderly is higher than in younger populations. The risk of developing HCC is higher among patients over 60 70 years old, and most elderly patients with HCC should have a background of cirrhosis. However, in this study, the proportion of cirrhosis among the elderly in the entire cohort was lower than younger patients $(64.1 \%$ vs $72.4 \%, P=0.111)$. This is probably related to patient selection during surgical decisionmaking.

Some tumor-related factors, such as preoperative AFP level $>400 \mu \mathrm{g} / \mathrm{L}$, satellite nodules, macrovascular invasion, and microvascular invasion as identified in the entire cohort of this study were independently associated with decreased OS and RFS after major hepatectomy for large HCC. They have also been demonstrated by previously reported studies to be high-risk factors. ${ }^{25-27}$ In addition, the surgery-related variable of intraoperative blood transfusion was also identified in this study to be independent risk factors associated with OS and RFS. A possible explanation is that allogenic blood transfusion induces immunosuppression and decreases natural-killer cell and/or 
Table 6 Univariable and Multivariable Cox-Regression Analyses of Risk Factors of Overall Survival After Major Hepatectomy for Large Hepatocellular Carcinoma in the 87elderly Patients

\begin{tabular}{|c|c|c|c|c|c|}
\hline Variables & HR Comparison & UV HR $(95 \% \mathrm{CI})$ & UV P & MV HR $(95 \% \mathrm{CI})$ & $M V P *$ \\
\hline Age & $\geq 75$ vs $65 \sim 74$ years & $0.77(0.31-1.93)$ & 0.578 & & \\
\hline Sex & Male vs Female & $0.74(0.36-1.50)$ & 0.406 & & \\
\hline Co-morbidities & Yes vs No & $1.13(0.56-2.30)$ & 0.727 & & \\
\hline ASA score & $>2$ vs $\leq 2$ & $1.12(0.67-1.86)$ & 0.660 & & \\
\hline Etiology of liver diseases & HBV vs Non-HBV & $2.21(1.19-4.10)$ & 0.012 & NA & 0.556 \\
\hline Cirrhosis & Yes vs No & $1.16(0.68-2.00)$ & 0.586 & & \\
\hline Portal hypertension & Yes vs No & $1.29(0.67-2.49)$ & 0.444 & & \\
\hline Child-Pugh grade & $B$ vs $A$ & $1.38(0.55-3.47)$ & 0.487 & & \\
\hline Preoperative AFP level & $>400$ vs $\leq 400 \mu \mathrm{g} / \mathrm{L}$ & $2.20(1.27-3.82)$ & 0.005 & $1.84(1.04-3.26)$ & 0.037 \\
\hline Maximum tumor size & $\geq 10$ vs $5.0-9.9 \mathrm{~cm}$ & $0.82(0.49-1.36)$ & 0.434 & & \\
\hline Multiple tumors ( $\geq 3$ nodules) & Yes vs No & $2.43(1.31-4.54)$ & 0.005 & $2.07(1.18-3.62)$ & 0.001 \\
\hline Satellite nodules & Yes vs No & $2.30(1.34-3.94)$ & 0.003 & NA & 0.750 \\
\hline Poor tumor differentiation & Yes vs No & $1.25(0.73-2.17)$ & 0.417 & & \\
\hline Macrovascular invasion & Yes vs No & $2.68(1.55-4.62)$ & $<0.001$ & $3.01(1.58-5.74)$ & 0.011 \\
\hline Microvascular invasion & Yes vs No & $1.76(1.04-2.97)$ & 0.036 & NA & 0.214 \\
\hline Incomplete tumor encapsulation & Yes vs No & $3.75(2.03-6.95)$ & $<0.001$ & NA & 0.245 \\
\hline Intraoperative blood loss & $>600$ vs $\leq 600 \mathrm{~mL}$ & $1.25(0.75-2.08)$ & 0.388 & & \\
\hline Intraoperative blood transfusion & Yes vs No & $1.14(0.69-1.89)$ & 0.609 & & \\
\hline Resection margin & $<\mathrm{I} v \mathrm{vs} \geq \mathrm{Icm}$ & $1.61(0.938-2.77)$ & 0.084 & NA & 0.341 \\
\hline
\end{tabular}

Notes: *All variables with a $P$ value $<0.1$ in univariable analysis were subjected to multivariable Cox-regression model.

Abbreviations: ASA, American Society of Anesthesiologists; HBV, hepatitis B virus; AFP, alpha-fetoprotein; Cl, confidence interval; HR, Hazard ratio; UV, univariable; MV, multivariable; NA, not available.

Table 7 Univariable and Multivariable Cox-Regression Analyses of Risk Factors of Recurrence-Free Survival After Major Hepatectomy for Large Hepatocellular Carcinoma in the 87 Elderly Patients

\begin{tabular}{|c|c|c|c|c|c|}
\hline Variables & HR Comparison & UV HR (95\% Cl) & UV P & MV HR $(95 \% \mathrm{Cl})$ & $M V P *$ \\
\hline Age & $\geq 75$ vs $65 \sim 74$ years & $0.68(0.27-\mid .7 I)$ & 0.415 & & \\
\hline Sex & Male vs Female & $0.69(0.36-\mid .3 I)$ & 0.257 & & \\
\hline Co-morbidities & Yes vs No & $0.94(0.46-1.89)$ & 0.855 & & \\
\hline ASA score & $>2$ vs $\leq 2$ & $0.90(0.56-1.47)$ & 0.685 & & \\
\hline Etiology of liver diseases & HBV vs Non-HBV & $2.39(1.35-4.23)$ & 0.003 & NA & 0.156 \\
\hline Cirrhosis & Yes vs No & $1.49(0.89-2.48)$ & 0.130 & & \\
\hline Portal hypertension & Yes vs No & $1.85(0.94-4.25)$ & 0.142 & & \\
\hline Child-Pugh grade & $B$ vs $A$ & I.76 (0.87-4.7I) & 0.201 & & \\
\hline Preoperative AFP level & $>400$ vs $\leq 400 \mu \mathrm{g} / \mathrm{L}$ & $1.81(1.08-3.03)$ & 0.026 & $1.82(1.03-3.22)$ & 0.040 \\
\hline Maximum tumor size & $\geq 10$ vs $5.0-9.9 \mathrm{~cm}$ & $0.85(0.53-1.37)$ & 0.512 & NA & 0.096 \\
\hline Multiple tumors ( $\geq 3$ nodules) & Yes vs No & $1.89(1.02-3.53)$ & 0.045 & $2.47(1.37-4.44)$ & 0.003 \\
\hline Satellite nodules & Yes vs No & $2.48(1.46-4.22)$ & 0.001 & & \\
\hline Poor tumor differentiation & Yes vs No & $1.68(0.99-2.88)$ & 0.056 & NA & 0.851 \\
\hline Macrovascular invasion & Yes vs No & $3.57(2.06-6.19)$ & $<0.001$ & $2.71(1.54-4.77)$ & 0.001 \\
\hline Microvascular invasion & Yes vs No & $2.15(1.31-3.55)$ & 0.003 & $1.85(1.06-3.21)$ & 0.029 \\
\hline Incomplete tumor encapsulation & Yes vs No & $3.21(1.84-5.57)$ & $<0.001$ & NA & 0.297 \\
\hline Intraoperative blood loss & $>600 \mathrm{vs} \leq 600 \mathrm{~mL}$ & $1.41(0.87-2.28)$ & 0.164 & & \\
\hline Intraoperative blood transfusion & Yes vs No & $1.21(0.75-1.96)$ & 0.428 & & \\
\hline Resection margin & $<\mathrm{I} v \mathrm{~s} \geq \mathrm{I} \mathrm{cm}$ & I.4I $(0.89-2.67)$ & 0.167 & & \\
\hline
\end{tabular}

Notes: *All variables with a $P$ value $<0.1$ in univariable analysis were subjected to multivariable Cox-regression model.

Abbreviations: ASA, American Society of Anesthesiologists; HBV, hepatitis B virus; AFP, alpha-fetoprotein; Cl, confidence interval; HR, hazard ratio; UV, univariable; MV, multivariable; NA, not available. 

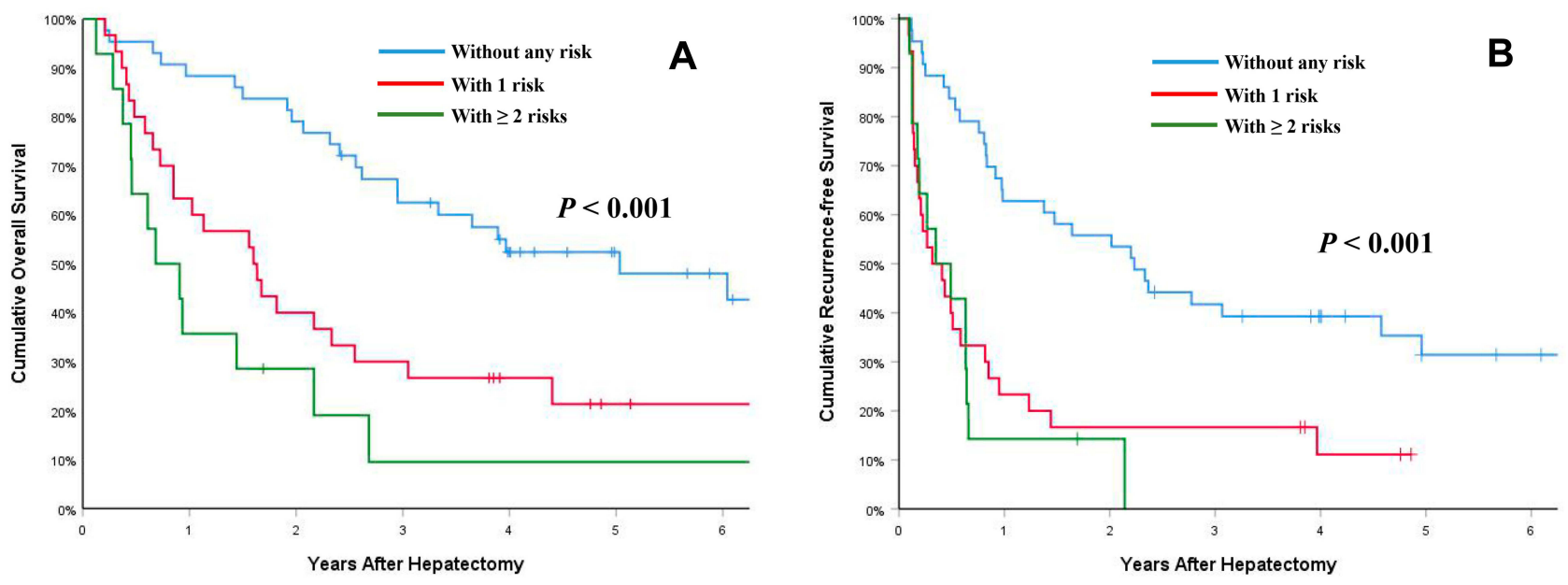

Figure 3 Cumulative incidence of overall survival (OS, 3A) and recurrence-free survival (RFS, 3B) curves comparisons among three subgroups of the elderly patients (calculated by Log-rank test).

helper T-cell activities. ${ }^{28}$ In fact, the relationship between blood transfusion and long-term prognosis after curative resection of many malignancies, including HCC, remains controversial. ${ }^{16,29}$ Anyway, avoidance of unnecessary blood transfusions is of importance for other reasons like saving costs, avoiding transfusion reactions and transfusion-related acute lung injury, and reducing risks of bloodtransmitting diseases.

In this study of 830 patients with large HCC, $25.6 \%$ had macrovascular invasion, $64.4 \%$ had microvascular invasion, $58.3 \%$ were huge $(\geq 10 \mathrm{~cm})$, and $19.6 \%$ were multiple tumors ( $\geq 3$ tumor nodules). Therefore, it is understandable that the recurrence rate of this cohort was higher than those of previous studies of this kind. As the tumor size increases, the probability of having other advanced malignant pathological characteristics, including macrovascular and microvascular invasion, multiformity, satellite nodules, and incomplete tumor encapsulation, will increase. As shown in Tables 3 and 4, univariable analyses of the entire cohort revealed that there were significant differences of OS and RFS between patients with HCCs of $5.0-9.9 \mathrm{~cm}$ and HCCs $\geq 10 \mathrm{~cm}$ (HR: 1.23 and 1.24, $P=$ 0.015 and 0.006 , respectively). However, multivariable Cox-regression analyses did not reveal maximum tumor size ( $\geq 10 \mathrm{vs} 5.0-9.9 \mathrm{~cm})$ as an independent risk factor of OS and RFS, but macrovascular invasion, microvascular invasion and satellite nodules as independent risk factors associated with poorer OS and RFS. In one previous study, for solitary hepatocellular carcinoma without macroscopic vascular invasion, we demonstrated that tumor size did not independently affect long-term OS and RFS after curative resection. ${ }^{30}$ As we think, although tumor size is also an important prognostic factor of $\mathrm{HCC}$ resection, its effect on a cohort of patients with large HCC $(\geq 5 \mathrm{~cm})$ may be somewhat weaker compared with other tumor characteristics, such as macrovascular and microvascular invasion, and satellite nodules, etc.

A deeper understanding of the risk factors associated with long-term oncological survival outcomes in elderly patients subjected to major hepatectomy for HCC can help surgeons on management, decision-making and determine strategies whether to give adjuvant treatment. In the present study, univariable and multivariable Cox-regression analysis performed in the elderly cohort identified four independent risk factors associated with OS and DFS. With the exception of microvascular invasion, these risk factors of preoperative AFP level, tumor number and macrovascular invasion can be identified by preoperative laboratory and imaging examinations. Interestingly, the 14 $(16.1 \%, 14$ of 87$)$ patients who had $\geq 2$ of these 3 independent risk factors had 1-year OS and RFS rates of only $35.7 \%$ and $14.3 \%$, respectively. Thus, for elderly patients with multiple risk factors, the benefit of major hepatectomy for large HCC is questionable. Future studies are required to better define how postoperative moleculartargeted and emerging immunotherapy drugs may be combined with hepatectomy to provide these patients with better survival outcomes.

This study has several limitations. First, this is a retrospective cohort study that had potential inherent biases. Second, patients in the present study were also treated exclusively in China and, therefore, the majority 
of patients with HCC had a background of HBV-related cirrhosis. In the United States and Europe, ${ }^{31,32} \mathrm{HCV}$ infection and excessive alcohol are the main etiological factors. As such, data from the present study need to be externally validated in Western patients to ensure whether the results are generalizability to other populations of patients.

\section{List of Abbreviations}

HCC, hepatocellular carcinoma; OS, overall survival; RFS, recurrence-free survival; ASA, American Society of Anesthesiologists; AFP, alpha-fetoprotein; CT, computed tomography; MRI, magnetic resonance imaging; HBV, hepatitis B virus; HR, hazard ratio; $\mathrm{CI}$, confidence interval; $\mathrm{HCV}$, hepatitis $\mathrm{C}$ virus; $\mathrm{MV}$, multivariable; NA, not available; UV, univariable; OR, odds ratio.

\section{Author Contributions}

Conception: Zheng-Liang Chen, Cheng-Wu Zhang, Tian Yang, Ying-Jian Liang, Wan Yee Lau; Study design: YingJian Liang, Zheng-Liang Chen, Cheng-Wu Zhang, Lei Liang, Feng Shen; Administrative support: Cheng-Wu Zhang, Meng-Chao Wu, Feng Shen, Ying-Jian Liang, Tian Yang; Data collection and acquisition: Zheng-Liang Chen, Lei Liang, Han Wu, Wan-Guang Zhang, Yong-Yi Zeng, WeiMin Gu, Ting-Hao Chen, Jie Li, Yao-Ming Zhang, Hong Wang, Ya-Hao Zhou, Chao Li, Yong-Kang Diao; Data analysis: Ying-Jian Liang, Zheng-Liang Chen, Tian Yang, YongKang Diao; Writing-original draft: Zheng-Liang Chen, Lei Liang, Han Wu, Wan-Guang Zhang, Yong-Yi Zeng, Wei-Min Gu, Ting-Hao Chen, Jie Li, Yao-Ming Zhang, Hong Wang, Ya-Hao Zhou, Chao Li, Yong-Kang Diao; Writing-review \& editing: Wan Yee Lau, Feng Shen, Meng-Chao Wu, Ying-Jian Liang, Tian Yang; Final approval of manuscript: All authors. All authors contributed to data analysis, drafting or revising the article, gave final approval of the version to be published, and agree to be accountable for all aspects of the work.

\section{Funding}

This work was supported in part by the National Natural Science Foundation of China (No. 81672699 and 81972726).

\section{Disclosure}

The authors report no conflicts of interest in this work.

\section{References}

1. Villanueva A. Hepatocellular carcinoma. N Engl J Med. 2019;380 (15):1450-1462. doi:10.1056/NEJMra1713263
2. El-Serag HB, Rudolph KL. Hepatocellular carcinoma: epidemiology and molecular carcinogenesis. Gastroenterology. 2007;132 (7):2557-2576. doi:10.1053/j.gastro.2007.04.061

3. Agrawal S, Belghiti J. Oncologic resection for malignant tumors of the liver. Ann Surg. 2011;253(4):656-665. doi:10.1097/SLA.0b013e $3181 \mathrm{fc} 08 \mathrm{ca}$

4. Müller-Höcker J, Aust D, Rohrbach H, et al. Defects of the respiratory chain in the normal human liver and in cirrhosis during aging. Hepatology. 1997;26(3):709-719. doi:10.1002/hep.510260324

5. Kaibori M, Yoshii K, Hasegawa K, et al. Treatment optimization for hepatocellular carcinoma in elderly patients in a Japanese nationwide cohort. Ann Surg. 2019;270(1):121-130. doi:10.1097/SLA.0000000 000002751

6. Mirici-Cappa F, Gramenzi A, Santi V, et al. Treatments for hepatocellular carcinoma in elderly patients are as effective as in younger patients: a 20-year multicentre experience. Gut. 2010;59(3):387-396. doi:10.1136/gut.2009.194217

7. Cucchetti A, Sposito C, Pinna AD, et al. Effect of age on survival in patients undergoing resection of hepatocellular carcinoma. Br J Surg. 2016;103(2):e9399. doi:10.1002/bjs.10056

8. Ezaki T, Yukaya H, Ogawa Y. Evaluation of hepatic resection for hepatocellular carcinoma in the elderly. Br $J$ Surg. 1987;74 (6):471-473. doi:10.1002/bjs. 1800740614

9. Nanashima A, Abo T, Nonaka T, et al. Prognosis of patients with hepatocellular carcinoma after hepatic resection: are elderly patients suitable for surgery. J Surg Oncol. 2011;104(3):284-291. doi:10. $1002 /$ jso. 21932

10. Zhao LY, Huo RR, Xiang X, et al. Hepatic resection for elderly patients with hepatocellular carcinoma: a systematic review of more than 17,000 patients. Expert Rev Gastroenterol Hepatol. 2018;12 (10):1059-1068. doi:10.1080/17474124.2018.1517045

11. Wong TC, Cheung TT, Chok KS, et al. Treatment strategy to improve long-term survival for hepatocellular carcinoma smaller than $5 \mathrm{~cm}$ : major hepatectomy vs minor hepatectomy. World J Surg. 2014;38 (9):2386-2394. doi:10.1007/s00268-014-2601-4

12. Sgourakis G, Sotiropoulos GC, Bockhorn M, et al. Major liver resections for primary liver malignancies in the elderly. Acta Chir Belg. 2009;109(3):340-344. doi:10.1080/00015458.2009.11680436

13. Wang WL, Zhu Y, Cheng JW, et al. Major hepatectomy is safe for hepatocellular carcinoma in elderly patients with cirrhosis. Eur $J$ Gastroenterol Hepatol. 2014;26(4):444-451. doi:10.1097/MEG.0 000000000000046

14. Yang T, Zhang J, Lu JH, Yang GS, Wu MC, Yu WF. Risk factors influencing postoperative outcomes of major hepatic resection of hepatocellular carcinoma for patients with underlying liver diseases. World J Surg. 2011;35(9):2073-2082. doi:10.1007/s00268-011-1161-0

15. Yang T, Lin C, Zhai J, et al. Surgical resection for advanced hepatocellular carcinoma according to Barcelona Clinic Liver Cancer (BCLC) staging. J Cancer Res Clin Oncol. 2012;138(7):1121-1129. doi:10.1007/s00432-012-1188-0

16. Yang T, Lu JH, Lau WY, et al. Perioperative blood transfusion does not influence recurrence-free and overall survivals after curative resection for hepatocellular carcinoma: a propensity score matching analysis. J Hepatol. 2016;64(3):583-593. doi:10.1016/j.jhep.2015.10.012

17. Dindo D, Demartines N, Clavien PA. Classification of surgical complications: a new proposal with evaluation in a cohort of 6336 patients and results of a survey. Ann Surg. 2004;240(2):205-213. doi:10.1097/01.sla.0000133083.54934.ae

18. Trevisani F, Cantarini MC, Labate AM, et al. Surveillance for hepatocellular carcinoma in elderly Italian patients with cirrhosis: effects on cancer staging and patient survival. Am J Gastroenterol. 2004;99 (8):1470-1476. doi:10.1111/j.1572-0241.2004.30137.x

19. Kozyreva ON, Chi D, Clark JW, et al. A multicenter retrospective study on clinical characteristics, treatment patterns, and outcome in elderly patients with hepatocellular carcinoma. Oncologist. 2011;16 (3):310-318. doi:10.1634/theoncologist.2010-0223 
20. Ishizawa T, Hasegawa K, Aoki T, et al. Neither multiple tumors nor portal hypertension are surgical contraindications for hepatocellular carcinoma. Gastroenterology. 2008;134(7):1908-1916. doi:10.1053/j. gastro.2008.02.091

21. Yau T, Tang VY, Yao TJ, Fan ST, Lo CM, Poon RT. Development of Hong Kong liver cancer staging system with treatment stratification for patients with hepatocellular carcinoma. Gastroenterology. 2014;146 (7):1691-1700.e3. doi:10.1053/j.gastro.2014.02.032

22. Imamura H, Matsuyama Y, Tanaka E, et al. Risk factors contributing to early and late phase intrahepatic recurrence of hepatocellular carcinoma after hepatectomy. J Hepatol. 2003;38(2):200-207. doi:10.1016/S0168-8278(02)00360-4

23. Calcinotto A, Kohli J, Zagato E, Pellegrini L, Demaria M, Alimonti A. Cellular senescence: aging, cancer, and injury. Physiol Rev. 2019;99 (2):1047-1078. doi:10.1152/physrev.00020.2018

24. Hong H, Wang Q, Li J, Liu H, Meng X, Zhang H. Aging, Cancer and Immunity. J Cancer. 2019;10(13):3021-3027. doi:10.7150/jca.30723

25. Yeh CN, Chen MF, Lee WC, Jeng LB. Prognostic factors of hepatic resection for hepatocellular carcinoma with cirrhosis: univariate and multivariate analysis. J Surg Oncol. 2002;81(4):195-202. doi:10. 1002/jso. 10178

26. Zhou L, Rui JA, Wang SB, et al. Clinicopathological features, post-surgical survival and prognostic indicators of elderly patients with hepatocellular carcinoma. Eur J Surg Oncol. 2006;32 (7):767-772. doi:10.1016/j.ejso.2006.03.050
27. Hanazaki K, Kajikawa S, Koide N, Adachi W, Amano J. Prognostic factors after hepatic resection for hepatocellular carcinoma with hepatitis $\mathrm{C}$ viral infection: univariate and multivariate analysis. $\mathrm{Am}$ $J$ Gastroenterol. 2001;96(4):1243-1250. doi:10.1111/j.1572-0241. 2001.03634.X

28. Cata JP, Wang H, Gottumukkala V, Reuben J, Sessler DI. Inflammatory response, immunosuppression, and cancer recurrence after perioperative blood transfusions. Br J Anaesth. 2013;110(5):690-701. doi:10.1093/ bja/aet068

29. Liu L, Wang Z, Jiang S, et al. Perioperative allogenenic blood transfusion is associated with worse clinical outcomes for hepatocellular carcinoma: a meta-analysis. PLoS One. 2013;8(5):e64261. doi:10.1371/journal. pone.0064261

30. Zhang H, Yuan SX, Dai SY, et al. Tumor size does not independently affect long-term survival after curative resection of solitary hepatocellular carcinoma without macroscopic vascular invasion. World J Surg. 2014;38(4):947-957. doi:10.1007/s00268-013-2365-2

31. Lau GK, Leung YH, Fong DY, et al. High hepatitis B virus (HBV) DNA viral load as the most important risk factor for HBV reactivation in patients positive for HBV surface antigen undergoing autologous hematopoietic cell transplantation. Blood. 2002;99 (7):2324-2330. doi:10.1182/blood.V99.7.2324

32. Goh GB, Chang PE, Tan CK. Changing epidemiology of hepatocellular carcinoma in Asia. Best Pract Res Clin Gastroenterol. 2015;29 (6):919-928. doi:10.1016/j.bpg.2015.09.007

\section{Publish your work in this journal}

Cancer Management and Research is an international, peer-reviewed open access journal focusing on cancer research and the optimal use of preventative and integrated treatment interventions to achieve improved outcomes, enhanced survival and quality of life for the cancer patient.
The manuscript management system is completely online and includes a very quick and fair peer-review system, which is all easy to use. Visit http://www.dovepress.com/testimonials.php to read real quotes from published authors. 